\title{
Circulating miR-660-5p is associated with no-reflow phenomenon in patients undergoing primary percutaneous coronary intervention
}

Jian-Wei zhang ( $\sim$ zhangjianwei_2003@sina.com )

Beijing Anzhen Hospital, Capital Medical University https://orcid.org/0000-0003-1707-8667

Cheng-Ping Hu

beijing anzhen hospital

Ying-Xin Zhao

beijing anzhen hospital

Ling-Jie He

beijing friendship hospital

Research article

Keywords: No-Reflow phenomenon, primary percutaneous coronary intervention, ST segment elevation myocardial infarction, miR-660-5p

Posted Date: June 17th, 2020

DOI: https://doi.org/10.21203/rs.3.rs-17913/v2

License: (c) (i) This work is licensed under a Creative Commons Attribution 4.0 International License. Read Full License 


\section{Abstract}

Background: The no-reflow phenomenon (NRP) is an important factor affecting the prognosis of patients with acute myocardial infarction undergoing primary percutaneous coronary intervention (PPCl). This study aims to investigate the association of circulating miR-660-5p with NRP in patients with ST segment elevation myocardial infarction (STEMI) undergoing PPCI.

Methods: Consecutive patients diagnosed with anterior STEMI within $12 \mathrm{~h}$ of pain onset were included in the study; in these patients, coronary angiography confirmed that the infarct-related artery was the left anterior descending (LAD) artery. Angiographic NRP was defined as a final TIMI flow of 2 or a final TIMI flow of 3 with a myocardial blush grade $(M B G)<2$. High miR-660-5p was defined as a value in the third tertile. The relationship of circulating miR-660-5p with NRP was assessed using Spearman correlation analysis and multiple linear regression analysis.

Results: Fifty-two eligible patients were finally included in this study (mean age: $56 \pm 12.4$ years, $>65$ years: $53.8 \%$, male: $76.9 \%$, and mean BMI: $26.3 \pm 3.5$ ). The incidence of NRP was $38.5 \%$. Circulating miR-660-5p was significantly related to the mean platelet volume (MPV). Patients were divided into tertiles by miR660-5p levels (Q1: $\leq 7.18, \mathrm{Q} 2:$ 7.18-11.31, Q3: > 11.31). Patients in the high microRNA-660-5p group had almost a 6-fold higher risk of NRP than those in the low microRNA-660-5p group [(odds ratio $(O R)=5.68$, $95 \%$ confidence interval ( $\mathrm{Cl}) 1.40-23.07, \mathrm{p}=0.015)$. When analysed by tertiles, consistent trends of an increasing relative odds of NRP were reported (OR1 for Q2 VS. Q1: 1.25, 95\% Cl: 0.27-5.73, p=0.77; OR2 for Q3 VS. Q1: 5.96, 95\% Cl: 1.33-26.66, p=0.02), even after multivariable adjustment. Receiver operating characteristic curve analysis demonstrated that the microRNA-660-5p level of 10.17 was the best cut-off level to predict the incidence of the no-reflow phenomenon in patients undergoing primary percutaneous coronary intervention with an area under the curve (AUC) of 0.768 (95\% Cl: 0.636-0.890).

Conclusion: Circulating miR-660-5p was significantly associated with NRP, and it may be a useful biomarker to predict the incidence of NRP in patients with STEMI undergoing PPCI.

\section{Background}

The China PEACE study has shown that the number of patients with acute myocardial infarction (AMI) undergoing primary percutaneous coronary intervention (PPCI) has increased significantly over the past 10 years in China. However, there was no significant improvement in in-hospital mortality in patients with $\mathrm{AMI}^{[1]}$. One of the important reasons for this poor prognosis was the occurrence of the no-reflow phenomenon (NRP) during PPCl. Nearly $40 \%$ of patients with ST segment elevation myocardial infarction (STEMI) did not obtain complete myocardial reperfusion by ST segment resolution or myocardial staining grading (MBG) analysis despite successful treatment of the culprit lesion ${ }^{[2-3]}$. NRP is a phenomenon in which effective blood perfusion cannot be obtained in ischaemic myocardium after opening of the occluded vessels combined with the existence of a mechanical obstruction such as a coronary dissection, embolism, thrombus, coronary spasm or stenosis. Previous studies have confirmed that NRP 
is closely associated with in-hospital mortality, malignant arrhythmias and heart failure ${ }^{[4-6]}$. The causes of NRP are extremely complex, and it is an urgent scientific problem to explore its pathogenesis and seek effective intervention.

MicroRNAs (miRNAs) are important posttranscriptional regulators of numerous biological processes including cell growth, proliferation, differentiation, and apoptosis ${ }^{[7]}$. Recently, many studies have reported that circulating microRNAs can be considered biomarkers in cardiovascular disease ${ }^{[8]}$. miR-660-5p, a member of the microRNA family, has been shown to be positively associated with adverse cardiovascular outcomes in patients with STEMI ${ }^{[9]}$. Overexpression of miR-660 increased the production of active platelets in vitro ${ }^{[10]}$, indicating a potential role in NRP. However, there are no reports on the correlation between circulating miR-660-5p and NRP during PPCl. The purpose of this study is to investigate the association of circulating miR-660-5p levels with the no-reflow phenomenon (NRP) in patients with STEMI undergoing PPCl.

\section{Methods}

From June 2017 to May 2018, we recruited patients diagnosed with anterior STEMI who were treated with $\mathrm{PPCl}$ within $12 \mathrm{~h}$ of pain onset. STEMI was diagnosed based on prolonged chest pain (>30 min) and ST segment elevation $>0.2 \mathrm{mv}$ in two or more adjacent leads ${ }^{[11]}$. Patients were finally included when coronary angiography confirmed that the infarct-related artery (IRA) was the left anterior descending artery. Patients who met the following criteria were excluded: age $>85$ years, previous myocardial infarction and $\mathrm{PCl}$ history, long-term oral anticoagulant drug treatment, acute infections, malignancy, severe liver and kidney dysfunction, chronic inflammatory diseases, and overt heart failure (Killip III or IV). All patients were treated with aspirin $(300 \mathrm{mg})$ and clopidogrel $(600 \mathrm{mg})$ on admission. The study was approved by the Anzhen Hospital Institutional Ethics Review Board, and all patients agreed to have their blood evaluated for scientific purposes.

The PPCl operation and perioperative medication administration were performed in accordance with the relevant clinical guidelines ${ }^{[12-13]}$. All PPCl operations were performed with 6- or 7-Fr guiding catheters through a transradial approach. A bolus of 70-100 IU/kg of heparin was given. Pre-dilatation was performed if necessary, and second-generation drug eluting stents were directly implanted whenever possible. The type of stent was determined by the operator. Only the LAD artery was treated, and non-IRAs were treated after 3 months if necessary. A glycoprotein Ilb/Illa inhibitor and thrombus aspiration device were used at the discretion of the operator. Intracoronary nitrate was always given after revascularization. We defined angiographic no-reflow as a final TIMI flow of 2 or a final TIMI flow of 3 with a myocardial blush grade $(\mathrm{MBG})<2^{[14]}$. The success of PPCl was defined as $<30 \%$ residual stenosis after recanalization of the LAD artery. High miR-660-5p was defined as a value in the third tertile.

Venous peripheral blood was collected before PPCl and centrifuged at $3500 \mathrm{~g}$ for $5 \mathrm{~min}$. After that, it was stored at $-80^{\circ} \mathrm{C}$ in a centrifuge tube without RNA enzymes. The total RNA was extracted using an Ultrapure RNA extraction kit (Cat\#CW0581, CWbio. Co. Ltd., China). A total of $5 \mu \mathrm{g}$ of RNA was subjected 
to $1 \%$ agarose gel electrophoresis to determine the RNA integrity. A total of $1 \mu \mathrm{g}$ of the original total RNA samples with a miRNA First Strand Synthesis Kit (Cat\# CW2141, CWbio. Co. Ltd., China) was used for reverse transcription. The upstream primer of miR-660-5p was 5-TACCCATTGCATATCGGAGT-3, the downstream primer was 5-GCCAACCGAGAAGATGATG-3, the upstream primer of U6 was 5GCTTCGGCAGCATCATACTAAATAAT3, the downstream primer was 5GCTTCACAATTGGCGTGCGTCATCATCATCAT3, and the internal parameter was U6. An ABI7500 fluorescence quantitative PCR instrument and miRNA Real-Time PCR Assay Kit (Cat\# CW2142, CWbio. Co. Ltd., China) were used for real-time quantitative PCR. The qPCR procedure was performed at $95^{\circ} \mathrm{C}$ for $10 \mathrm{~min}$ followed by 40 cycles of $95^{\circ} \mathrm{C}$ for $15 \mathrm{~s}$ and $60^{\circ} \mathrm{C}$ for $60 \mathrm{~s}$. The operation was performed according to the manufacturer's protocol ${ }^{[15]}$. The $2^{-\triangle \Delta C T}$ method was used to calculate the relative expression of RNA samples.

\section{Statistical analysis}

Continuous data are displayed as the mean \pm standard deviation (SD). Groups of continuous data were compared by one-way analysis of variance (ANOVA) or the Kruskal-Wallis $H$ test. The association between circulating miR-660-5p and other parameters was determined by Pearson's test or Spearman's test. Multivariable logistic regression analysis was applied to verify the association of circulating miR$660-5 p$ with NRP. Variables with an unadjusted $p$-value $<0.1$ in the univariate analysis that were known to have a significant effect on NRP were entered into the multivariate model. All statistical analyses were performed using SPSS 24.0 software (IBM Corp., Armonk, NY, USA). A P-value $<0.05$ was required for statistical significance, and all tests were two-tailed.

\section{Results}

Fifty-two eligible patients were included in this study. Table 1 shows the baseline characteristics of patients according to the tertiles of miR-660-5p levels. The mean age was 56 years $₫$ standard deviation (SD): $12.4 \rrbracket$. Approximately $53.8 \%$ of patients were older than 65 years, and $76.9 \%$ of them were male. The mean BMI was $26.3 \mathrm{~kg} / \mathrm{m}^{2}$ (SD: 3.5 ). The incidence of NRP was $38.5 \%$. Patients were divided into tertiles by miR-660-5p levels (Q1: $\leq 7.18, \mathrm{Q} 2:$ 7.18-11.31, Q3: > 11.31). A high miR-660-5p level was defined as a value in the third tertile (>11.31), while a low microRNA-660-5p level was defined as a value in the lower two tertiles $(\leq 11.31)$. Compared with the lower tertile group, patients in the higher tertile group had a significantly higher mean platelet volume (MPV). There was no significant difference between the other variables. Table 2 displays the characteristics of coronary artery lesions and the procedural characteristics of PPCl. Ninety-four percent of patients with TIMI 0/1 grade on admission were admitted. Since $53.8 \%$ of the patients used tirofiban, only 2 patients underwent thrombus aspiration. Nearly half of the target lesions were at the proximal LAD artery. The incidence of angiographic NRP was $38.5 \%$. Patients with higher microRNA-660-5p levels had a significantly higher incidence of NRP (Figure 1).

Table 1 Baseline characteristics on admission according to microRNA-660-5p tertiles 


\begin{tabular}{|c|c|c|c|c|c|}
\hline & All (52) & $\mathrm{Q} 1(\mathrm{n}=17)$ & $\mathrm{Q} 2(\mathrm{n}=18)$ & $\mathrm{Q} 3(\mathrm{n}=17)$ & $P$ value \\
\hline Age (years) & $56 \pm 12.4$ & $59 \pm 12.6$ & $51.7 \pm 11.4$ & $56.9 \pm 12.6$ & 0.175 \\
\hline Age (口65years), n (\%) & $28(53.8)$ & $11(64.7)$ & $8(44.4)$ & $9(52.9)$ & 0.539 \\
\hline Male, n (\%) & $40(76.9)$ & $13(76.5)$ & $14(77.8)$ & $13(76.5)$ & - \\
\hline $\mathrm{BMI} \square \mathrm{kg} / \mathrm{m} 2$ & $26.3 \pm 3.5$ & $25.6 \pm 2.5$ & $27.2 \pm 3.5$ & $26.1 \pm 4.3$ & 0.424 \\
\hline Smokers, n (\%) & $39(75)$ & 13(76.5) & $14(77.8)$ & $12(70.6)$ & 0.924 \\
\hline Hypertension, n (\%) & $28(53.8)$ & $11(64.7)$ & $8(44.4)$ & $9(52.9)$ & 0.539 \\
\hline Systolic blood pressure (mmHg) & $120 \pm 16.9$ & $119 \pm 14.9$ & $119 \pm 16.3$ & $121 \pm 20.0$ & 0.949 \\
\hline Diastolic blood pressure (mmHg) & $77 \pm 12.7$ & $75 \pm 11.0$ & $76 \pm 12.6$ & $79 \pm 14.7$ & 0.626 \\
\hline Hypercholesterolaemia, n (\%) & $24(46.2)$ & $9(52.9)$ & $8(44.4)$ & $7(41.2)$ & 0.832 \\
\hline diabetes mellitus, n (\%) & $10(19.2)$ & $5(29.4)$ & $3(16.7)$ & $2(11.8)$ & 0.463 \\
\hline History of stroke/TIA, n (\%) & $9(17.3)$ & $5(29.4)$ & $2(11.1)$ & $2(11.8)$ & 0.422 \\
\hline \multicolumn{6}{|l|}{ KILLIP, n (\%) } \\
\hline 口 & $19 \square 36.5 \square$ & $4 \square 23.5 \square$ & $8 \square 44.4 \square$ & $7 \square 36.5 \square$ & 0.448 \\
\hline 吅 & $33 \llbracket 63.5 \square$ & $13 \square 76.5 \square$ & $10 \square 55.6 \square$ & $10 \square 63.5 \square$ & \\
\hline \multicolumn{6}{|l|}{ Laboratory examination } \\
\hline BNPmax & $1322 \pm 1797.6$ & $1781 \pm 2103.8$ & $597 \pm 508.8$ & $1632 \pm 2163.6$ & 0.102 \\
\hline FBG & $7.1 \pm 2.7$ & $7.5 \pm 3.7$ & $6.5 \pm 1.7$ & $7.3 \pm 2.7$ & 0.519 \\
\hline TG & $1.6 \pm 1.1$ & $1.8 \pm 1.4$ & $1.5 \pm 0.8$ & $1.6 \pm 0.9$ & 0.512 \\
\hline LDL-C & $2.7 \pm 0.8$ & $2.5 \pm 0.8$ & $2.7 \pm 0.8$ & $2.9 \pm 0.8$ & 0.311 \\
\hline HDL & $1.0 \pm 0.2$ & $0.9 \pm 0.1$ & $1.0 \pm 0.2$ & $1.0 \pm 0.2$ & 0.155 \\
\hline TCHO & $4.3 \pm 1.2$ & $4.1 \pm 1.2$ & $4.3 \pm 1.2$ & $4.6 \pm 1.0$ & 0.586 \\
\hline HGB & $141.8 \pm 18.5$ & $139.5 \pm 20.3$ & $143.6 \pm 22.5$ & $142 \pm 11.7$ & 0.806 \\
\hline Creatine & $59.4 \pm 14.9$ & $61 \pm 17.6$ & $57.9 \pm 12.9$ & $58.6 \pm 14.3$ & 0.744 \\
\hline D-dimer & $0.2 \pm 0.2$ & $0.1 \pm 0.1$ & $0.2 \pm 0.2$ & $0.1 \pm 0.1$ & 0.529 \\
\hline WBC (109 L-1) & $11.2 \pm 3.3$ & $11.2 \pm 3.2$ & $11.4 \pm 3.7$ & $11.1 \pm 3.3$ & 0.984 \\
\hline PLT (109 L-1) & $237 \pm 57.7$ & $249 \pm 57.8$ & $236 \pm 65.1$ & $225.5 \pm 49.7$ & 0.502 \\
\hline MPV & $12.0 \pm 2.3$ & $11.1 \pm 2.3$ & $11.3 \pm 1.7$ & $13.7 \pm 2.1$ & 0.001 \\
\hline TnT peak (ng/mL) & $48.4 \pm 15.5$ & $48.3 \pm 16.4$ & $50.6 \pm 13.1$ & $46 \pm 17.4$ & 0.697 \\
\hline
\end{tabular}




\begin{tabular}{llllll} 
CK-MB peak $(\mathrm{ng} / \mathrm{mL})$ & $247.5 \pm 209.9$ & $261.4 \pm 181.46$ & $202.7 \pm 131.4$ & $281.1 \pm 292.3$ & 0.524 \\
\hline CRP & $8.6 \pm 13.9$ & $13.0 \pm 21.2$ & $4 \pm 6.2$ & $8.9 \pm 8.7$ & 0.157 \\
\hline LVEF (\%) & $55.2 \pm 7.3$ & $53.8 \pm 8.07$ & $55.2 \pm 6.9$ & $56.5 \pm 7.1$ & 0.568 \\
\hline Medication & & & & - \\
\hline Statin, n (\%) & $52 \square 100 \square$ & $17 \square 100 \square$ & $18 \square 100 \square$ & $17 \square 100 \square$ & 0.765 \\
\hline Beta-blocker, n (\%) & $49 \square 94.2 \square$ & $16 \square 94.1 \square$ & $16 \square 88.9 \square$ & $17 \square 100 \square$ & 0.1 \\
\hline Ace-I/ARB, n (\%) & $42 \square 80.8 \square$ & $11 \square 64.7 \square$ & $15 \square 83.3 \square$ & $16 \square 94.1 \square$ & $10 \pm 2.9$ \\
\hline Duration of hospitalization(days) & $9 \pm 2.8$ & $9 \pm 2.5$ & $9 \pm 2.9$ & $16 \square 94.1 \square$ & 0.037 \\
\hline high MPV & $37 \square 71.2 \square$ & $10 \square 58.8 \square$ & $11 \square 61.1 \square$ & $16 \square$ \\
\hline
\end{tabular}

BMI: Body Mass Index; TIA: transient ischemic attacks; BNP: brain natriuretic peptide;FBG: fasting blood glucose; TG: Triglyceride; LDL-C: Triglyceride; HDL: High density lipoprotein; TCHO: Total cholesterol; HGB: Haemoglobin; WBC, white blood cell count; PLT, platelet count; MPV: Mean platelet volume; TnT peak, Troponin T peak ;CK-MB: creatine kinase-MB; CRP: C-reactive protein; LVEF: left ventricular ejection fraction; ACEI: Angiotensin-Converting Enzyme Inhibitors; ARB: angiotensin receptor blocker; Q1: $\leq$ 7.18, Q2: 7.18-11.31, Q3: $\square 11.31$.

Table 2 Coronary artery lesions and procedural characteristics according to microRNA-660-5p tertiles

\begin{tabular}{lrrrrr}
\hline & All patient(52) & Q1(n=17) & Q2(n=18) & Q3(n=17) & P value \\
\hline syntax & $16 \pm 4.5$ & $18 \pm 5.3$ & $14 \pm 3,7$ & $15 \pm 3.7$ & 0.02 \\
\hline Proximal-LAD & $26 \square 50 \square$ & $8 \llbracket 47.1 \square$ & $9 \square 50 \square$ & $9 \square 52.9 \square$ & - \\
\hline tirofiban infusion, n (\%) & $28 \square 53.8 \square$ & $10(58.8)$ & $7(38.9)$ & $11(64.7)$ & 0.304 \\
\hline Pain to balloon time (min) & $120.6 \pm 53.1$ & $119 \pm 48.9$ & $118 \pm 53.3$ & $124 \pm 59.5$ & 0.95 \\
\hline Thrombus-aspirating device usage, n (\%) & $2(3.8)$ & $1 \square 5.9 \square$ & $1 \square 5.6 \square$ & $0 \square 0 \square$ & - \\
\hline Pre-dilatation,n(\%) & $46(88.5)$ & $15(88.2)$ & $16(88.8)$ & $15(88.2)$ & - \\
\hline Stent implanted,n(\%) & $47(90.4)$ & $16(94.1)$ & $15(83.3)$ & $16(94.1)$ & 0.6 \\
\hline TIMI 0/1, n (\%) & $49(94.2)$ & $16(94.1)$ & $17(94.4)$ & $16(94.1)$ & - \\
\hline MBG0/1 & $19(36.5)$ & $5(29.4)$ & $4(22.2)$ & $10(36.5)$ & 0.689 \\
\hline Post-TIMI 4 & $9(17.3)$ & $1(5.9)$ & $1(5.6)$ & $7(41.2)$ & 0.01 \\
\hline
\end{tabular}


LAD: left anterior descending coronary artery; MBG: myocardial blush grade; Q1: $\leq 7.18$, Q2: 7.18-11.31,

Q3: $\square 11.31$

As shown in Table 3, patients with higher microRNA-660-5p levels had a higher risk of NRP than those with lower microRNA-660-5p levels [odds ratio $(\mathrm{OR})=1.30,95 \%$ confidence interval $(\mathrm{CI})$ 1.10-1.56, $p=0.003]$. In the multivariable analysis, microRNA-660-5p remained an independent predictor of angiographic NR (OR=1.34, 95\% Cl 1.10-1.63, $p=0.004)$. Patients with higher microRNA-660-5p levels had an almost 6-fold higher risk of coronary no-reflow than those with lower microRNA-660-5p levels $(\mathrm{OR}=5.68,95 \% \mathrm{Cl} 1.40$ to $23.07, p=0.015)$. When analysed by tertiles, consistent trends of increasing relative odds of angiographic NR were reported (OR1 for Q2 VS. Q1: 1.25, 95\% Cl: 0.27-5.73, p=0.77; OR2 for Q3 VS. Q1: 5.96, 95\% Cl: 1.33-26.66, $p=0.02)$.

Table 3 Relationship between microRNA-660-5p and coronary no-reflow

\begin{tabular}{|c|c|c|c|c|c|c|}
\hline & \multicolumn{2}{|c|}{ univariable analysis } & \multirow[b]{2}{*}{$P$ value } & \multicolumn{2}{|c|}{ multivariable analysis } & \multirow[b]{2}{*}{$P$-value } \\
\hline & OR & $95 \% \mathrm{CI}$ & & adjustedOR & $95 \% \mathrm{CI}$ & \\
\hline PLT & 0.99 & $0.98-1.00$ & 0.125 & 0.99 & $0.98-1.04$ & 0.212 \\
\hline LVEF & 0.98 & 0.908-1.06 & 0.624 & 0.97 & $0.89-1.06$ & 0.48 \\
\hline TnT peak & 0.99 & $0.96-1.03$ & 0.742 & 0.99 & $0.94-1.03$ & 0.504 \\
\hline tirofiban infusion, n (\%) & 1.5 & $0.48-4.65$ & 0.483 & 1.15 & $0.27-4.84$ & 0.852 \\
\hline Pain to balloon time (min) & 0.99 & $0.98-1.00$ & 0.27 & 0.99 & $0.98-1.01$ & 0.213 \\
\hline microRNA-660-5p & 1.3 & $1.10-1.56$ & 0.003 & 1.34 & $1.10-1.63$ & 0.004 \\
\hline High microRNA-660-5p & 5.3 & $1.52-18.50$ & 0.009 & 5.68 & $1.40-23.07$ & 0.015 \\
\hline Q1 & - & - & - & - & - & - \\
\hline Q2 & 1.25 & $0.27-5.73$ & 0.77 & 1.3 & $0.25-6.67$ & 0.752 \\
\hline Q3 & 5.96 & $1.33-26.66$ & 0.02 & 6.52 & $1.24-34.16$ & 0.027 \\
\hline
\end{tabular}

OR: odds ratio; CI: confidence interval; PLT, platelet count; LVEF: left ventricular ejection fraction; TnT peak,

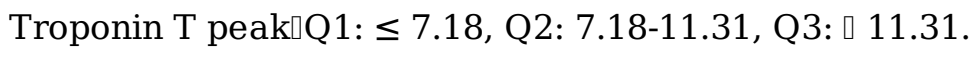

We also observed a significant correlation between miRNA-660-5p and MPV $(P<0.001)$, while there was no correlation with other markers measured on admission (Table 4, Figure 2).

Table 4 Correlation between admission microRNA-660-5p and other parameters 


\begin{tabular}{ccc}
\hline & $\mathrm{r}$ & $P$ Value \\
\hline PLT & -0.85 & 0.549 \\
\hline MPV & 0.567 & 0.0001 \\
\hline WBC & 0.068 & 0.633 \\
\hline CRP & 0.045 & 0.749 \\
\hline
\end{tabular}

PLT, platelet count; MPV: Mean platelet volume; WBC, white blood cell count; CRP: C-reactive protein.

\section{Discussion}

To our knowledge, this is the first study to report that miR-660-5p is an independent predictor of NRP in patients with STEMI undergoing PPCl. Moreover, we also found that miR-660-5p was closely related to MPV. Many studies have shown that several microRNAs may be involved in coronary plaque rupture and local thrombus formation. Since serum is easily measured, microRNAs may serve as disease biomarkers of $A M{ }^{[16]}$. miR-660-5p may have promise as a biomarker of NRP.

miR-660-5p has been shown to be significantly associated with adverse cardiovascular outcomes (cardiogenic death or recurrent myocardial infarction) in patients with STEMI, and it is an independent predictor of major adverse cardiovascular events (MACE) in patients with STEMI ${ }^{\left[{ }^{9]}\right.}$. However, the pathogenetic mechanisms, particularly how to regulate the no-reflow phenomenon, remain unclear.

Although the pathophysiology of NRP is not completely understood, several theories including reperfusion injury, distal thromboembolism with $\mathrm{PCl}$, microvascular arterial spasm, and endothelial dysfunction have been proposed ${ }^{[17]}$. It is well recognized that platelets play a key role in the occurrence of NRP. As platelet turnover is increased in STEMI, newly formed platelets with transient expression of an inducible COX-2 enzyme may be released into the circulation, leading to thromboxane A2 production in amounts sufficient to initiate platelet aggregation ${ }^{[18]}$. Several studies have shown that there is a significant correlation between platelet aggregation and coronary microvascular blood flow reduction [19]. This increased platelet volume has been linked to impaired reperfusion and more frequent NRP in patients treated with PPCl ${ }^{[20]}$. Oxidative stress has also been linked to the development of microvascular obstruction. Specifically, sustained levels of NOX2, the catalytic subunit of NADPH oxidase that is released by platelet activation, result in a vicious cycle of platelet aggregate stabilization and thrombus growth that contributes to $\mathrm{CNR}^{[21]}$. Huczek et al. found that patients with higher platelet reactivity assessed by PFA-100 had a significantly higher percentage of angiographic NRP ${ }^{[22]}$. Aurigemma $\mathrm{C}$ et al. reported that platelet activation in patients with coronary artery microvascular occlusion (MVO) was significantly higher than that in patients with complete myocardial reperfusion [23]. Thromboxane A2 (TXA2) is not only an important medium for platelet activation and aggregation but also an important medium for platelet-induced coronary artery contraction. Giampaolo Niccoli et al. thought that the plasma 
TXA2 level was an independent predictor of coronary angiography NR and lack of ST segment resolution after PPCI ${ }^{[24]}$. miR-660-5p has been reported to increase the production of active platelets in experimental studies ${ }^{[10]}$. Moreover, miR-660-5p has a positive effect on megakaryopoiesis and the output of activated platelets ${ }^{[25]}$. miR-660-5p has been speculated to regulate 2644 high confidence targets ${ }^{[26]}$, among which genes of the "enzyme-linked receptor signal pathway", "HDAC class I mediated signal event" and "glycosphingolipid biosynthesis" were significantly enriched. Nineteen genes including GATA1, TAL1, TESC and IL-11, which are well-known regulators of megakaryocyte differentiation, are considered to be regulatory genes of megakaryopoiesis. Overexpression of miR-660-5p can make the colony forming unit (CFU)-MKs increase by 3.2 times and the percentage of hyperploid cells increase from $4 \%$ in the control group to $11 \%$ in the overexpression group ${ }^{[10]}$. Although miR-660-5p could not increase the total number of cells, it increased the proportion of polyploid cells and the number of activated platelets. Thus, microRNA-660-5p may play a role in NFP with increased production of active platelets.

Moreover, we also found that miR-660-5p was closely related to MPV. The incidence of NRP after PPCI was significantly higher in patients with a high MPV ( $\geq 10.3 \mathrm{fl})$ than in patients with a low MPV $(<10.3 \mathrm{fl})$ ${ }^{[20]}$. Furthermore, MPV could be an independent predictor of coronary no-reflow and mortality. Larger platelets are more active in metabolism and enzymes than smaller platelets. Several studies have shown that larger platelets produce more prothrombotic factors and more dense granules and aggregate preferentially and more rapidly ${ }^{[27]}$.

In summary, these findings suggest that circulating miR-660-5p is of value in NRP. miR-660-5p may play a role in NRP with increased production of active platelets and MPV. It may be considered for early evaluation of NRP in PPCl. Moreover, these findings indicate that incorporating circulating miR-660-5P levels into clinical decision making has the potential to guide treatment more accurately. Therefore, further studies to identify the optimal cut-off values and to clarify possible other mechanisms are necessary.

There were some limitations in this study. First, the sample size was small; thus, larger sample size, multicentre, prospective studies are needed to confirm our results. Second, the lack of collection and analysis of miR-660-5p levels in a normal population may make our study less rigorous. Third, the potential mechanism of the correlation between miR-660-5p and NRP has not been elucidated and needs to be fully clarified through further basic research.

\section{Conclusion}

In conclusion, circulating miR-660-5p was significantly associated with NRP in patients with STEMI undergoing PPCl, and it may be a useful biomarker to predict the incidence of NRP in patients with STEMI undergoing PPCl.

\section{List Of Abbreviations}


AMI: acute myocardial infarction; PPCl: primary percutaneous coronary intervention ;NRP: no-reflow phenomenon; STEMI: ST segment elevation myocardial infarction ;MBG: myocardial staining grading; miRNAs: MicroRNAs; IRA: infarct-related artery; MACE: major adverse cardiovascular events; BMI: Body Mass Index; TIA: transient ischemic attacks; BNP: brain natriuretic peptide; FBG: fasting blood glucose; TG: Triglyceride; LDL-C: Triglyceride; HDL: High density lipoprotein; TCHO: Total cholesterol; HGB: Hemoglobin; WBC, white blood cell count; PLT, platelet count; MPV: Mean platelet volume; TnT peak, Troponin T peak ;CK-MB: creatine kinase-MB; CRP: C-reactive protein; LVEF: left ventricular ejection fraction; ACEI: Angiotensin-Converting Enzyme Inhibitors; ARB: angiotensin receptor blocker; OR: odds ratio; $\mathrm{Cl}$ : confidence interval

\section{Declarations}

\section{Ethics approval and consent to participate}

This study was approved by anzhen Hospital institutional medical ethical committee. All patients provided written informed consent.

\section{Consent for publication}

Not applicable.

\section{Availability of data and materials}

The datasets analyzed during the current study are available from the corresponding author on reasonable request.

\section{Competing interests}

The authors declare that they have no competing interests.

\section{Fundings}

This work were supported by the Beijing Municipal Administration of Hospitals Clinical Medicine Development of Special Funding Support (code: ZYLX201303『XMLX201601)区the grant from National Key Research and Development Program of China (2017YFC0908800)凶Beijing Municipal Administration of Hospitals' Ascent Plan (Code: DFL20150601) and Mission plan (Code: SML20180601)

\section{Authors' contributions}

Jianwei Zhang and Lingjie He designed the experiments; Jianwei Zhang and chengping hu performed the experiments; Jianwei Zhang and Yingxin Zhao analyzed the experimental results and wrote the manuscript. Both authors read and approved the final manuscript.

\section{Acknowledgements}


The authors thank the staff at the Department of cardiology, the Beijing Anzhen hospital of Capital Medical University, and all the patients who participated in the study.

\section{References}

[1] Li J, Li X, Wang Q, Hu S, Wang Y, Masoudi FA, et al. ST-segment elevation myocardial infarction in China from 2001 to 2011 (the China PEACE-Retrospective Acute Myocardial Infarction Study): a retrospective analysis of hospital data. Lancet. 2015; 385:441-51.

[2] Galasso G, Schiekofer S, D'Anna C, Gioia GD, Piccolo R, Niglio T, et al. No-Reflow Phenomenon: Pathophysiology, Diagnosis, Prevention, and Treatment. A Review of the Current Literature and Future Perspectives. Angiology. 2014; 65:180-9.

[3] The Lancet. 40 years of percutaneous coronary interven-tion: Where next? Lancet. 2017; 390: 715

[4] Eeckhout E, Kern MJ. The coronary no-reflow phenomenon: a review of mechanisms and therapies. Eur Heart J. 2001; 22:729-39.

[5] Stone GW, Peterson MA, Lansky AJ, Dangas G, Meenran R, Leon MB. Impact of normalized myocardial perfusion after successful angioplasty in acute myocardial infarction. J Am Coll Cardiol. 2002; 39: 591-7

[6] Resnic FS, Wainstein M, Lee MK, Behrendt D, Wainstein RV, Ohno-Machado L, et al. No-reflow is an independent predictor of death and myocardial infarction after percutaneous coronary intervention. Am Heart J. 2003; 145: 42-6

[7] Bartel DP. MicroRNAs: genomics, biogenesis, mechanism, and function. Cell. 2004; 116: 281-97.

[8] Wang C, Jing Q. Non-coding RNAs as biomarkers for acute myocardial infarction. Acta Pharmacol Sin. 2018; 39: 1110-9.

[9] Jakob P, Kacprowski T, Briand-Schumacher S, Heg D, Klingenberg R, Stähli BE, et al. Profiling and validation of circulating microRNAs for cardiovascular events in patients presenting with ST-segment elevation myocardial infarction. Eur Heart J. 2017; 38:511.

[10] Emmrich S, Henke K, Hegermann J, Ochs M, Reinhardt D, Klusmann JH. miRNAs can increase the efficiency of ex vivo platelet generation. Ann Hematol. 2012; 91:1673-84.

[11] Thygesen K, Alpert JS, Jaffe AS, Chaitman BR, Bax JJ, Morrow DA, et al. Fourth Universal Definition of Myocardial Infarction. Circulation. 2018;138: e618-e651.

[12] Ibanez B, James S, Agewall S, Antunes MJ, Bucciarelli-Ducci C, Bueno H, et al. 2017 ESC Guidelines for the management of acute myocardial infarction in patients presenting with ST-segment elevation: The Task Force for the management of acute myocardial infarction in patients presenting with ST-segment elevation of the European Society of Cardiology (ESC). Eur Heart J. 2018; 39:119-77. 
[13] Neumann FJ, Sousa-Uva M, Ahlsson A, Alfonso F, Banning AP, Benedetto U, et al. 2018 ESC/EACTS Guidelines on myocardial revascularization. Eur Heart J. 2019; 40: 87-165.

[14] Gibson CM, Murphy SA, Morrow DA, Aroesty JM, Gibbons RJ, Gourlay SG, et al. Angiographic perfusion score: an angiographic variable that integrates both epicardial and tissue level perfusion before and after facilitated percutaneous coronary intervention in acute myocardial infarction. Am Heart J. 2004; 148:336-40

[15] Bustin SA, Benes V, Garson JA, Hellemans J, Huggett J, Kubista M, et al. The MIQE guidelines: minimum information for publication of quantitative real-time PCR experiments. Clin Chem. 2009; 55:61122.

[16] Fung EC, Butt AN, Eastwood J, Swaminathan R, Sodi R. Circulating microRNA in cardiovascular disease. Adv Clin Chem, 2019, 91: 99-122.

[17] Karimianpour A, Maran A. Advances in coronary no-reflow phenomenon-a contemporary review. Curr Atheroscler Rep. 2018, 20: 44

[18] Rocca B, Secchiero P, Ciabattoni G, Ranelletti FO, Catani L, Guidotti L, et al. Cyclooxygenase-2 expression is induced during human megakaryopoiesis and characterizes newly formed platelets. Proc Natl Acad Sci U S A. 2002; 99:7634-9.

[19] Chaitman B, Lim B. No reflow and the quest to achieve optimal perfusion during the acute phase of myocardial infarction. J Am Coll Cardiol. 2004; 44:313-5.

[20] Huczek Z, Kochman J, Filipiak KJ, Horszczaruk GJ, Grabowski M, Piatkowski R, et al. Mean platelet volume on admission predicts impaired reperfusion and long-term mortality in acute myocardial infarction treated with primary percutaneous coronary intervention. J Am Coll Cardiol. 2005; 46:284-90.

[21] Niccoli G, Celestini A, Calvieri C. Patients with microvascular obstruction after primary percutaneous coronary intervention show a gp91 phox (NOX2) mediated persistent oxidative stress after reperfusion. Eur Heart J Acute Cardiovasc Care. 2013; 2:379-88.

[22] Huczek Z, Filipiak KJ, Kochman J, Piatkowski R, Grabowski M, Roik M, et al. Baseline platelet reactivity in acute myocardial infarction treated with prymari angioplasty-influence on myocardial reperfusion, left ventricular performance, and clinical events. Am Heart J. 2007; 154:62-70

[23] Aurigemma C, Scalone G, Tomai F, Altamura L, De Persio G, Stazi A, et al. Persistent enhanced platelet activation in patients with acute myocardial infarction and coronary microvascular obstruction: clinical implications. Thromb Haemost. 2014; 111:122-30.

[24] Niccoli G, Giubilato S, Russo E, Spaziani C, Leo A, Porto I, et al. Plasma levels of thromboxane A2 on admission are associated with no-reflow after primary percutaneous coronary intervention. Eur Heart J. 2008; 29:1843-50. 
[25] Garzon R, Pichiorri F, Palumbo T, Iuliano R, Cimmino A, Aqeilan R, et al. MicroRNA fingerprints during human megakaryocytopoiesis. Proc Natl Acad Sci U S A.2006; 103:5078-83

[26] Xiao F, Zuo Z, Cai G, Kang S, Gao X, Li T. miRecords: an integrated resource for microRNA-target interactions. Nucleic Acids Res.2009; 37: D105-10.

[27] Martin J, Bath PMW, Burr ML. Increased platelet size following myocardial infarction is associated with subsequent death and nonfatal reinfarction. Lancet. 1991; 338:1409-11

\section{Figures}

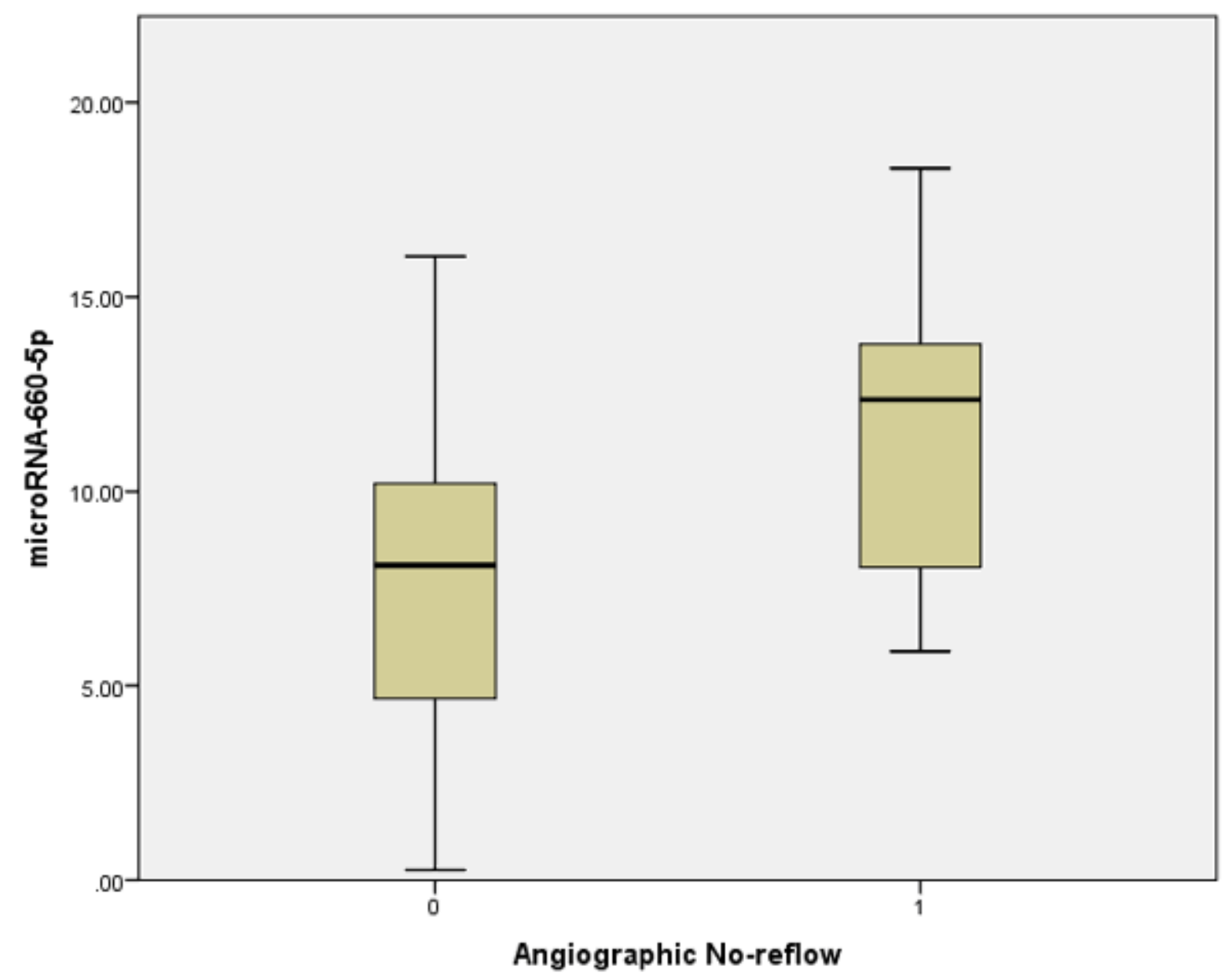

\section{Figure 1}

Comparison of miR-660-5p levels according to angiographic no-reflow phenomenon 


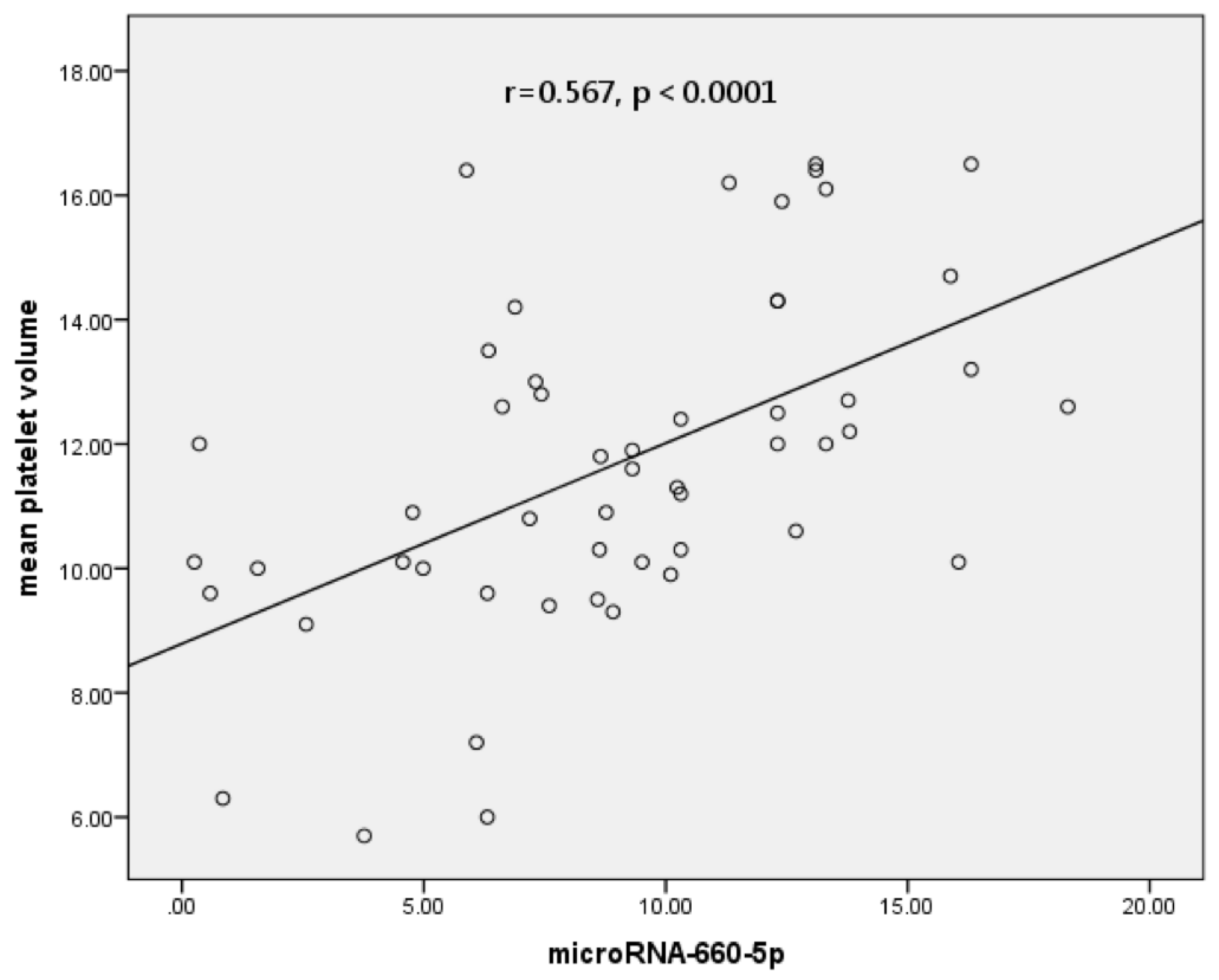

Figure 2

Correlation between miR-660-5p levels and mean platelet volume 


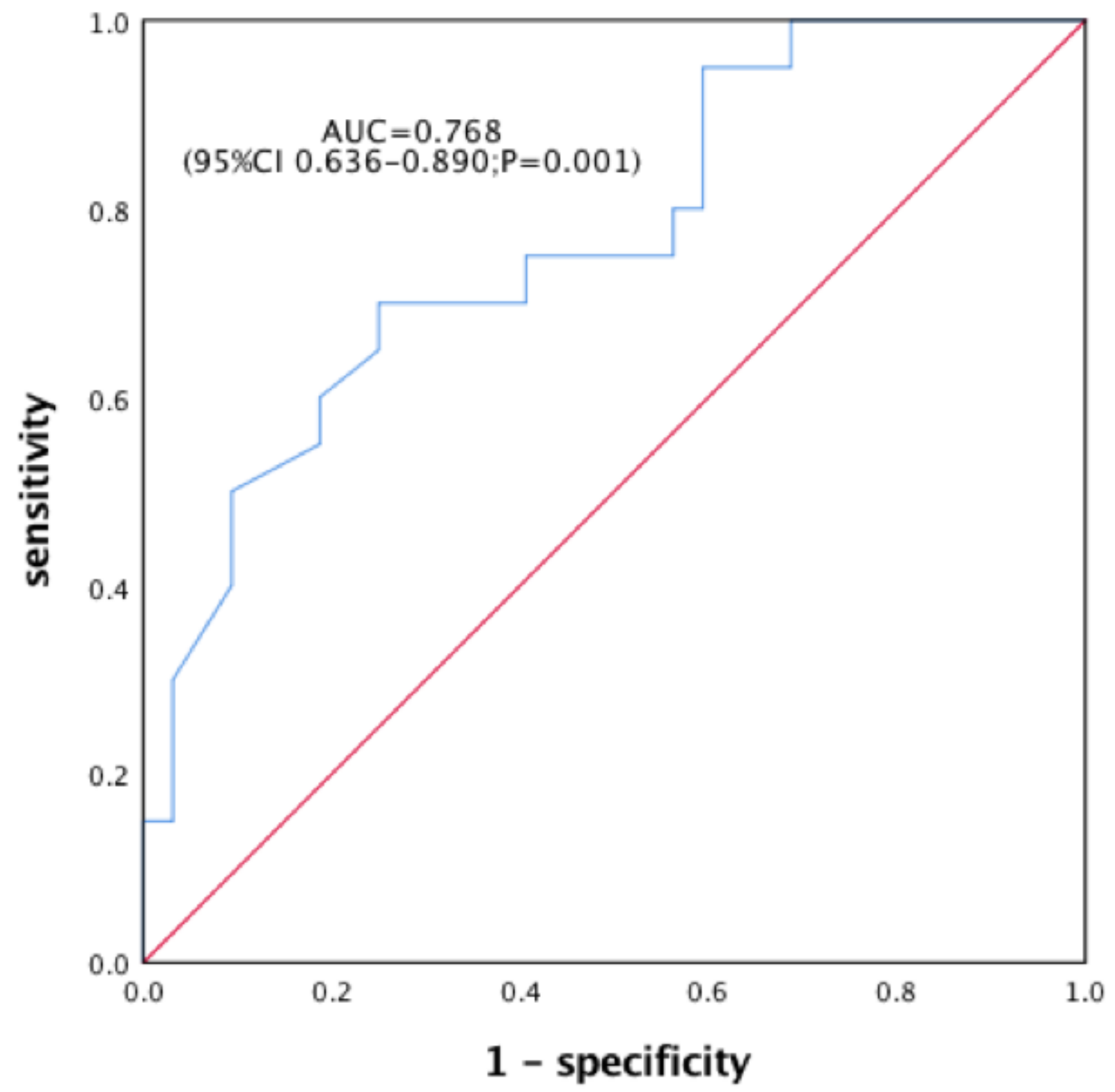

Figure 3

Receiver operating characteristic (ROC) curves of microRNA-660-5p as a marker to predict no-reflow phenomenon 